\title{
Post-exposure effect of music through personal listening device (PLD) on speech perception in noise test in young adults
}

\author{
Original \\ Article \\ Naema Mohamed Ismail', Ebtesam Hamed Nada ${ }^{2}$, Reda Mohamed Behairy, Mona Taha \\ Abdel Haleem Hegazy ${ }^{4}$
}

${ }^{1}$ Department of ENT, Faculty of Medicine, Al Azhar University, Cairo, ${ }^{2} Z a g a z i g$ University, Zagazig, ${ }^{3}$ Lecturer of Audiovestibular Medicine, Faculty of Medicine for Girls, Al-Azhar

University, ${ }^{4}$ Al Azhar Teaching Hospital, Cairo, Egypt.

\begin{abstract}
Introduction: Tinnitus is often perceived temporarily after noise exposure, usually disappearing within a few hours. However, a significant number of young people perceive permanent tinnitus which is the most frequent symptom following exposure to noise via PLDs.

Objective: To evaluate the effect of music exposure through (PLD) with and without tinnitus on results of EHFA, TEOAEs, ABR and SPIN tests in normal hearing young adults.

Patients and Methods: Sixty normal hearing young adults were included in this study, fifteen as a control group and forty-five PLDs users as study group, the study group were subdivided according to the presence of tinnitus into two subgroups. All subjects were submitted to basic audiological evaluation (pure tone audiometry, speech audiometry and immittancemetry), Extended high frequency audiometry (EHFA), transient evoked otoacoustic emissions (TEOAEs), auditory brainstem response (ABR) and speech perception in noise (SPIN) test.

Results: There were statistically significant differences in extended high frequency audiometry (HFA) at different frequencies among study and control groups. There were statistically significant differences in transient evoked otoacoustic emission test (TEOAEs) response among control and study groups. There were no statistically significant differences in absolute and inter-peak latencies of low and high repetition rate ABR among control and studied groups. There were statistically significant differences in speech perception in noise test (SPIN) among the control and study groups.

Conclusion: : exposure to recreational noise due to excessive PLDs usage cause elevated EHFA thresholds, decreased TEOAEs amplitudes and decreased SPIN scores despite normal conventional PTA thresholds.
\end{abstract}

Key Words: Music, noise, tinnitus.

Received: 01 July 2019, Accepted: 02 March 2020

Corresponding Author: Reda Mohamed Behairy, Department of ENT, Lecturer of Audiovestibular Medicine, Faculty of Medicine for Girls, Al Azhar University, Cairo, Egypt. Tel.: 01222739249, E-mail: drredabehairy@yahoo.com

ISSN: 2090-0740, July 2020 Vol.21, No. 2

\section{INTRODUCTION}

Tinnitus is hearing sound when no external sound is present ${ }^{[1]}$. It is often described as a ringing. It may also sound like a clicking, hiss or roaring. Rarely, unclear voices or music are heard. The sound may be soft or loud, low pitched or high pitched and appear to be coming from one ear (unilateral) or both (bilateral). Most of the time, it comes on gradually. In some people, tinnitus causes depression, anxiety or interferes with concentration ${ }^{[2]}$.

Although tinnitus is usually associated with hearing loss, it also may occur with normal hearing because normal hearing thresholds do not necessarily indicate the absence of cochlear damage or complete organization of central auditory system ${ }^{[3,4]}$.

The several background noises that are present in daily life can sometimes make listening difficult, especially when trying to understand speech. So, Speech-in-Noise tests are designed to indicate the ability to understand speech in presence of background noise. The test contains sentences that simulate a range of contextual situations encountered in everyday speech communication. The evaluation of SPIN test should be considered a very important aspect to be measured in human auditory function ${ }^{[5]}$. Some studies reported the effect of tinnitus on speech reception in noise ${ }^{[6]}$. showed that a group of hearing impaired tinnitus patients had significantly worse speech perception abilities compared to a control group with the same hearing impairment levels. Tinnitus patients with normal hearing often complain of a reduced ability to communicate and discriminate between words, especially in noisy environments ${ }^{[7]}$. However, it has not been determined whether the speech perception ability of these patients is actually reduced by internal noise or by 
other causes as hidden HL or central deficit ${ }^{[8]}$.

Most of teenagers and young adults use personal listening devices (PLDs) such as mp3 players and mobile phones. The availability of portable PLDs with long battery life, large storage capacity and high quality of sound output has allowed users to regularly listen to these devices for long time and at high volumes ${ }^{[9]}$. They are at risk to develop noise-induced symptoms such as hearing loss, hyperacusis, and tinnitus which is the most frequently symptom reported by PLDs users ${ }^{[12]}$. Some studies found no measurable peripheral damage in tinnitus subjects with normal hearing at conventional audiometry. Gilles et al. ${ }^{[10]}$ suggested that the presence of tinnitus in PLD users may occur without measurable peripheral damage and might cause more central reorganization at the level of cochlear nuclei than expected. Another study found no peripheral affection in tinnitus subjects with normal conventional PTA (no significant difference between tinnitus and nontinnitus subjects as regard EHFA and OAEs ${ }^{[1]}$.

However, many studies proved the presence of peripheral damage (in the form of decreased TEOAEs or elevated EHFA thresholds) in tinnitus subjects with normal hearing at conventional audiograms. Fernandes and dos Santos, ${ }^{[12]}$ found that the TEOAE S/N ratio values were lower in the tinnitus group than the tinnitus-free group on all analyzed frequencies and for both left and right ears. Also, Paglialonga et al. ${ }^{[13]}$ showed decreased TEOAE S/N ratio in tinnitus subjects with normal hearing sensitivity ${ }^{[14,15,16]}$ compared EHFA in tinnitus patients with normal hearing to age and gender matched controls and reported hearing loss for extended high-frequency audiometry in tinnitus subjects.A controversy exists in adults with normal hearing thresholds and tinnitus due to leisure noise, so this study is designed to assess the differences in audiological characteristics between noiseexposed adults with and without tinnitus.

\section{PATIENTS AND METHODS}

This study was conducted during the period from $15 / 03 / 2017$ to $20 / 04 / 2018$ after Approval of Ethical Committee at 8/02/2017. Subjects included in the present study were divided into two groups:

a) Control Group: Included 15 normal healthy subjects not using PLDs regularly (collected from relatives and preemployment evaluation) matched the study group as regard age and gender.

b) Study Groups:Included 45 subjects, they subdivided into two subgroups:

- Group 1 (G1): consisted of 30 subjects with history of regular exposure to music through PLDs and complaining of tinnitus,referred from ENT clinic.
- Group 2 (G2): consisted of 15 subjects with history of regular exposure to music through PLDs but without complaining of tinnitus, randomly selected from university students.

Both control and study groups had bilateral normal peripheral hearing (with hearing threshold levels not exceeding $25 \mathrm{~dB}$ at any frequency in the range of $250-8000 \mathrm{~Hz})$ and age range (18-45 years). In the present study all study group were PLDs users used to prefer listening to tonal music (songs) during their daily travelling which is around 2-3 hour per day and set the sound volume at the maximum level of the device with average exposure period of 3 months and was examined 2 days after last exposure to avoid tinnitus of temporary period $^{[10]}$.

\section{Exclusion criteria:}

Subjects with the following criteria were excluded from the study:

1. Age below 18 or above 45 years.

2. Objective or somatic tinnitus.

3. Middle ear pathology.

4. Congenital ear malformation.

5. Any neurologic diseases.

6. Hearing loss.

7. Diabetes, hypertension, trauma and tumors.

8. Tinnitus accompanied by vertigo, history of ototoxic drug use, or hearing loss with a conductive or surgically correctible component.

9. Occupational noise exposure.

10. Retro-cochlear lesions.

\section{B-Methods:}

All subjects in this study were submitted to the following:

1. Full History taking.

2. Otological examination.

3. Basic audiological evaluation including: -Pure tone audiometry (PTA) for both air and bone conduction and Speech audiometry using Madsen, Orbiter 922. -Immittancemetry using Madsen Zodiac 901 which include: a) Tympanometry which was done at varying pressureranging from +200 to $-400 \mathrm{mmH} 2 \mathrm{O}$ to evaluate themiddle ear pressure and compliance and to exclude middle ear pathology.

b) Acoustic reflex thresholds elicited both ipsilateraly and contralateraly using pure tones of 500, 1000, 2000 and $4000 \mathrm{~Hz}$. 
4. Extended high frequency audiometry including: -10 , 12.5 and $16 \mathrm{kHz}$ by using Sennheiser HDA 200 circumaural head phones.

5. Otoacoustic Emissions: Transient evoked otoacoustic emissions (TEOAEs):

TEAOE amplitudes were measured in both ears of subjects using a diagnostic OAE analyser (Otodynamics ILO V6). During the tests, subjects were seated comfortably in a sound-attenuated booth and told to limit their movements. An OAE probe fitted with appropriatesized ear tip was placed in the external ear canal of subjects and the probe fit was confirmed with the in-the-ear calibration by the software.

TEOAEs were elicited using non-linear click sounds of $100 \mu$ s presented at an intensity level of $80 \mathrm{dBSPL}$ with 260 presentations of click and recorded over a frequency band of $1000-4000 \mathrm{~Hz}$. The stability of the stimulus was maintained at $\geq 80 \%$ and reproducibility level of $\geq 70 \%{ }^{[17]}$. The overall amplitude of the TEOAE spectrum and TEOAE amplitudes at five frequency bands $(1,1.4,2,2.8$ and $4 \mathrm{kHz})$ were determined. OAEs were considered as present when the signal to noise ratio (SNR) exceeded $3 \mathrm{~dB}$ and were considered as absent when the SNR was below $2.99 \mathrm{~dB}^{[18]}$.

(6) Auditory brain stem response (ABR) as neurootologic evaluation:

ABR were recorded in a sound-treated room with a BioLogic Navigator Pro interface. The skin was prepared by use of a Nuprep gel in order to lower the skin impedance which had to be below $5 \mathrm{KOhm}$. Electrodes were placed on both mastoids and on the forehead. Subjects lay down on a comfortable bed and the light was subdued. Subjects were also instructed to keep the eyes closed during the measurements and to minimize all muscle activity as much as possible.

1024 rarefaction acoustic clicks each of $100 \mu \mathrm{sec}$ duration were presented monaurally via supra aural earphones at an intensity of $90 \mathrm{dBnHL}$ between 2000$4000 \mathrm{~Hz}$ at low repetition rate (LRR) and high repetition rate (HRR) (21.1 and $71 \mathrm{p} \backslash \mathrm{sec}$.). The band pass filter was set at 100 to $1500 \mathrm{~Hz}$ and the recording sensitivity was adjusted to 31.67 microvolts.

Identifiable and repeatable ABR waves (I, III, V) were detected when either ear was stimulated at $90 \mathrm{dBnHL}$. Both absolute and inter-peak latencies were calculated as well.

(7) Arabic speech perception in noise (SPIN) test: Arabic Speech in noise Test (SPIN) was done using Arabic Phonetically Balanced (PB) words recorded in a back ground of cafeteria noise. It consisted of 8 lists each of 25 monosyllabic words. The speech test materials and noise were delivered monaurally to each ear through headphones. The patient was asked to repeat the words and ignore the noise. The stimulus was presented at a level of 45-50 dB SL with a signal to noise ratio (SNR) equal zero $\mathrm{dB}$ meaning that speech and noise are presented equally loud (45-50 dB SL). Scoring was done by calculating the number of $\%$ correct words out of the total ${ }^{[19]}$.

\section{Statistical analysis}

Data were analyzed by Statistical Package of Social Science (SPSS), software version 24.0 (SPSS Inc., 2016).Continuous data were presented as the Mean \pm SD if normally distributed or Median (Range) if not normally distributed. Normality was checked by Shapiro test.Categorical data were presented by the count and percentage.The one-way analysis of variance (ANOVA): is used to determine whether there are any significant differences between the means of two or more independent (unrelated) groups on a continuous dependent variable.

Tukey post hoc test: it is used for multiple comparisons between groups following ANOVA test. The chi-squared test: is used to discover if there is a relationship between two categorical variables.

$P$-value $<0.05$ indicates significant, $P<0.01$ indicates highly significant difference, $P<0.001$ indicates very highly significant difference while, $P \geq 0.05$ indicates nonsignificant difference .

\section{RESULTS}

No statistically significant differences between control and studied groups as regards age and gender (table 1).No statistically significant differences in pure tone audiometry (PTA) at different frequencies among control and studied groups (table 2).Recreational noise with tinnitus (G1) and recreational noise without tinnitus (G2) groups had higher EHFA thresholds at all frequencies bilaterally from (10 to $16 \mathrm{KHz}$ ) than control group with statistically significant differences (table 3). Recreational noise with tinnitus (G1) and recreational noise without tinnitus (G2) groups had lower TEOAEs at all frequency bands bilaterally from $(1000$ to $4000 \mathrm{~Hz}$ ) and total OAE response than control group with statistically significant differences (table 4). No statistically significant differences in absolute and inter-peak latencies of low repetition rate $\mathrm{ABR}$ among control and studied groups (table 5), no statistically significant differences in absolute and inter-peak latencies of high repetition rate $\mathrm{ABR}$ among control and studied groups (table 6). Highly statistically significant differences in speech perception in noise test (SPIN) was found between the studied groups and controls in both ears (table 7). 
Table 1: Demographic data of the studied groups

\begin{tabular}{lccccc}
\hline Variables & $\begin{array}{c}\mathrm{CG} \\
\mathrm{n}=15\end{array}$ & $\begin{array}{c}\mathrm{G} 1^{*} \\
\mathrm{n}=30\end{array}$ & $\begin{array}{c}\mathrm{G} 2^{*} \\
\mathrm{n}=15\end{array}$ & Significance test & P-value \\
\hline $\begin{array}{l}\text { Age (years) } \\
\text { Mean } \pm \mathrm{SD}\end{array}$ & $29.5 \pm 7.4$ & $29.4 \pm 6.3$ & $28 \pm 5.1$ & $\begin{array}{c}\text { ANOVA } \\
(\mathrm{F})=0.29\end{array}$ & 0.75 \\
$\begin{array}{l}\text { Gender } \\
\text { Male, n (\%) }\end{array}$ & $7(47 \%)$ & $16(53 \%)$ & $7(47 \%)$ & & 0.87 \\
Female, n $(\%)$ & $8(53 \%)$ & $14(47 \%)$ & $8(53 \%)$ & $\chi 2=0.27$ & \\
\hline
\end{tabular}

$\chi 2$, Chi-squared test

*CG: control group.

*G1 subjects with music exposur through PLDs with tinnitus group.

$*^{*}$ 22: subjects with music exposur through PLDs without tinnitus group.

Table 2: Comparison between control and studied groups as regard to pure tone audiometry (PTA) thresholds.

\begin{tabular}{|c|c|c|c|c|c|c|}
\hline Frequency/ Hz & Side & $\begin{array}{c}\mathrm{CG} \\
\mathrm{n}=15 \\
\text { Mean } \pm \mathrm{SD}\end{array}$ & $\begin{array}{c}\mathrm{G} 1 \\
\mathrm{n}=30 \\
\mathrm{Mean} \pm \mathrm{SD}\end{array}$ & $\begin{array}{c}\mathrm{G} 2 \\
\mathrm{n}=15 \\
\mathrm{Mean} \pm \mathrm{SD}\end{array}$ & $\begin{array}{c}\text { ANOVA } \\
\mathrm{F}=\end{array}$ & $P$-value \\
\hline \multirow{2}{*}{250} & RT & $14.2 \pm 3.5$ & $14.9 \pm 3.8$ & $14.7 \pm 4.3$ & 0.17 & 0.85 \\
\hline & $\mathrm{LT}$ & $14.3 \pm 3.1$ & $15 \pm 3.6$ & $15.3 \pm 3.9$ & 0.32 & 0.73 \\
\hline \multirow{2}{*}{500} & $\mathrm{RT}$ & $13 \pm 2.4$ & $13.7 \pm 3.3$ & $14.4 \pm 3.7$ & 0.71 & 0.49 \\
\hline & LT & $12.7 \pm 3.1$ & $13.2 \pm 2.7$ & $13.3 \pm 2.9$ & 0.20 & 0.82 \\
\hline \multirow{2}{*}{1000} & RT & $11 \pm 2.7$ & $12.2 \pm 2.6$ & $12.6 \pm 2.5$ & 1.60 & 0.21 \\
\hline & $\mathrm{LT}$ & $11.3 \pm 2.2$ & $11.7 \pm 2.4$ & $12 \pm 3.1$ & 0.29 & 0.75 \\
\hline \multirow{2}{*}{2000} & RT & $10 \pm 3.2$ & $11.2 \pm 2.1$ & $10.7 \pm 1.7$ & 1.33 & 0.27 \\
\hline & LT & $10.3 \pm 2.9$ & $11.5 \pm 2.3$ & $11.8 \pm 2.8$ & 1.49 & 0.23 \\
\hline \multirow{2}{*}{4000} & RT & $10.5 \pm 1.8$ & $12 \pm 3$ & $12.3 \pm 3.1$ & 1.92 & 0.16 \\
\hline & LT & $10.2 \pm 2.8$ & $12.5 \pm 3.4$ & $12.4 \pm 3.3$ & 2.76 & 0.07 \\
\hline \multirow{2}{*}{8000} & RT & $11.1 \pm 4.2$ & $11.9 \pm 3.6$ & $12.1 \pm 3.8$ & 0.31 & 0.74 \\
\hline & LT & $11.4 \pm 3.9$ & $13.6 \pm 3.2$ & $13.8 \pm 2.2$ & 2.87 & 0.06 \\
\hline
\end{tabular}

*CG: control group, G1 subjects with music exposur through PLDs with tinnitus.

${ }^{*} \mathrm{G} 2$ : subjects with music exposur through PLDs without tinnitus. 
Table 3: Comparison between control and studied groups as regard to extended high frequency audiometry (EHFA) thresholds.

\begin{tabular}{|c|c|c|c|c|c|c|c|}
\hline Frequency/ Hz & Side & $\begin{array}{c}\mathrm{CG} \\
\mathrm{n}=15 \\
\text { Mean } \pm \text { SD }\end{array}$ & $\begin{array}{c}\mathrm{G} 1 \\
\mathrm{n}=30 \\
\text { Mean } \pm \text { SD }\end{array}$ & $\begin{array}{c}\mathrm{G} 2 \\
\mathrm{n}=15 \\
\text { Mean } \pm \text { SD }\end{array}$ & $\begin{array}{c}\text { ANOVA } \\
\mathrm{F}=\end{array}$ & $P$-value & Tukey post hoc test \\
\hline \multirow{2}{*}{10000} & RT & $14.2 \pm 3.5$ & $14.9 \pm 3.8$ & $14.7 \pm 4.3$ & 0.17 & 0.85 & $\begin{array}{l}\text { CG vs. } \mathrm{G} 1=0.035 \\
\mathrm{CG} \text { vs. } \mathrm{G} 2=0.013\end{array}$ \\
\hline & $\mathrm{LT}$ & $14.3 \pm 3.1$ & $15 \pm 3.6$ & $15.3 \pm 3.9$ & 0.32 & 0.73 & $\begin{array}{l}\mathrm{CG} \text { vs. } \mathrm{G} 1=0.015 \\
\mathrm{CG} \text { vs. } \mathrm{G} 2=0.007\end{array}$ \\
\hline \multirow{2}{*}{12500} & RT & $13 \pm 2.4$ & $13.7 \pm 3.3$ & $14.4 \pm 3.7$ & 0.71 & 0.49 & $\begin{array}{l}\text { CG vs. } \mathrm{G} 1=0.034 \\
\mathrm{CG} \text { vs. } \mathrm{G} 2=0.011\end{array}$ \\
\hline & $\mathrm{LT}$ & $12.7 \pm 3.1$ & $13.2 \pm 2.7$ & $13.3 \pm 2.9$ & 0.20 & 0.82 & $\begin{array}{l}\mathrm{CG} \text { vs. } \mathrm{G} 1=0.006 \\
\mathrm{CG} \text { vs. } \mathrm{G} 2=0.001\end{array}$ \\
\hline \multirow{2}{*}{16000} & RT & $11 \pm 2.7$ & $12.2 \pm 2.6$ & $12.6 \pm 2.5$ & 1.60 & 0.21 & $\begin{array}{l}\text { CG vs. } \mathrm{G} 1=0.045 \\
\mathrm{CG} \text { vs. } \mathrm{G} 2=0.003\end{array}$ \\
\hline & $\mathrm{LT}$ & $11.3 \pm 2.2$ & $11.7 \pm 2.4$ & $12 \pm 3.1$ & 0.29 & 0.75 & $\begin{array}{l}\mathrm{CG} \text { vs. } \mathrm{G} 1=0.032 \\
\mathrm{CG} \text { vs. } \mathrm{G} 2=0.005\end{array}$ \\
\hline
\end{tabular}

*CG: control group.

G1 subjects with music exposur through PLDs with tinnitus.

*G2: subjects with music exposur through PLDs without tinnitus..

Table 4: Comparison between control and studied groups as regard to transient evoked otoacoustic emission test (TEOAEs) at different frequencies and total OAE response.

\begin{tabular}{|c|c|c|c|c|c|c|c|}
\hline Frequency/ Hz & Side & $\begin{array}{c}\mathrm{CG} \\
\mathrm{n}=15 \\
\text { Mean } \pm \mathrm{SD}\end{array}$ & $\begin{array}{c}\mathrm{G} 1 \\
\mathrm{n}=30 \\
\text { Mean } \pm \text { SD }\end{array}$ & $\begin{array}{c}\mathrm{G} 2 \\
\mathrm{n}=15 \\
\text { Mean } \pm \text { SD }\end{array}$ & $\begin{array}{c}\text { ANOVA } \\
\mathrm{F}=\end{array}$ & $P$-value & Tukey post hoc test \\
\hline \multirow{2}{*}{10000} & RT & $13.7 \pm 5.1$ & $10.8 \pm 3$ & $10.3 \pm 2.4$ & 4.4 & 0.017 & $\begin{array}{l}\text { CG vs. } \mathrm{G} 1=0.031 \\
\text { CG vs. } \mathrm{G} 2=0.028\end{array}$ \\
\hline & $\mathrm{LT}$ & $14.3 \pm 5.2$ & $10.4 \pm 3.1$ & $10.5 \pm 2.5$ & 6.4 & 0.003 & $\begin{array}{l}C G \text { vs. } G 1=0.003 \\
C G \text { vs. } G 2=0.015\end{array}$ \\
\hline \multirow{2}{*}{1400} & RT & $14 \pm 5.5$ & $10.6 \pm 3.4$ & $10.1 \pm 1.4$ & 5.3 & 0.008 & $\begin{array}{l}\text { CG vs. } \mathrm{G} 1=0.014 \\
\mathrm{CG} \text { vs. } \mathrm{G} 2=0.015\end{array}$ \\
\hline & $\mathrm{LT}$ & $14.1 \pm 5.3$ & $10.7 \pm 3.3$ & $9.9 \pm 1.5$ & 6.1 & 0.004 & $\begin{array}{l}\text { CG vs. } \mathrm{G} 1=0.011 \\
\mathrm{CG} \text { vs. } \mathrm{G} 2=0.006\end{array}$ \\
\hline \multirow{2}{*}{2000} & RT & $18.8 \pm 4.1$ & $15.2 \pm 4.2$ & $14.7 \pm 3.2$ & 5.2 & 0.009 & $\begin{array}{l}\text { CG vs. } G 1=0.015 \\
C G \text { vs. } G 2=0.017\end{array}$ \\
\hline & LT & $19 \pm 3.7$ & $14.8 \pm 4.6$ & $13.8 \pm 4.3$ & 6.5 & 0.003 & $\begin{array}{l}\text { CG vs. } \mathrm{G} 1=0.009 \\
\text { CG vs. } \mathrm{G} 2=0.005\end{array}$ \\
\hline \multirow{2}{*}{2800} & RT & $18.5 \pm 4.1$ & $14.9 \pm 4.5$ & $14.6 \pm 2.8$ & 4.7 & 0.013 & $\begin{array}{l}\text { CG vs. G1 }=0.018 \\
\text { CG vs. G2 }=0.028\end{array}$ \\
\hline & $\mathrm{LT}$ & $18.7 \pm 4$ & $14.5 \pm 3.5$ & $13.9 \pm 4.4$ & 7.4 & 0.001 & $\begin{array}{l}\text { CG vs. G1 }=0.003 \\
\text { CG vs. } G 2=0.004\end{array}$ \\
\hline \multirow{2}{*}{4000} & RT & $16.1 \pm 4.2$ & $12.7 \pm 3.9$ & $12.1 \pm 2.9$ & 5.3 & 0.008 & $\begin{array}{l}\text { CG vs. } \mathrm{G} 1=0.016 \\
\text { CG vs. } \mathrm{G} 2=0.014\end{array}$ \\
\hline & LT & $17.8 \pm 5.0$ & $13.2 \pm 3.8$ & $12.4 \pm 4.7$ & 7.2 & 0.002 & $\begin{array}{l}\text { CG vs. } \mathrm{G} 1=0.004 \\
\mathrm{CG} \text { vs. } \mathrm{G} 2=0.004\end{array}$ \\
\hline \multirow{2}{*}{ Total OAE } & RT & $11.8 \pm 1.7$ & $10 \pm 1.9$ & $9.8 \pm 2.1$ & 5.5 & 0.007 & $\begin{array}{l}\text { CG vs. } \mathrm{G} 1=0.011 \\
\text { CG vs. } \mathrm{G} 2=0.015\end{array}$ \\
\hline & LT & $12.3 \pm 1.6$ & $10.2 \pm 2.2$ & $9.7 \pm 2$ & 7.4 & 0.001 & $\begin{array}{l}\mathrm{CG} \text { vs. } \mathrm{G} 1=0.005 \\
\mathrm{CG} \text { vs. } \mathrm{G} 2=0.002\end{array}$ \\
\hline
\end{tabular}

*CG: control group.

G1 subjects with music exposur through PLDs with tinnitus.

*G2: subjects with music exposur through PLDs without tinnitus. 
Table 5: Comparison between control and studied groups as regard to absolute and inter-peak latencies (IPL) of LRR ABR.

\begin{tabular}{|c|c|c|c|c|c|c|}
\hline Latencies & Side & $\begin{array}{c}\mathrm{CG} \\
\mathrm{n}=15 \\
\text { Mean } \pm \text { SD }\end{array}$ & $\begin{array}{c}\mathrm{G} 1 \\
\mathrm{n}=30 \\
\text { Mean } \pm \text { SD }\end{array}$ & $\begin{array}{c}\mathrm{G} 2 \\
\mathrm{n}=15 \\
\text { Mean } \pm \text { SD }\end{array}$ & $\begin{array}{c}\text { ANOVA } \\
\mathrm{F}=\end{array}$ & $P$-value \\
\hline \multirow{2}{*}{ Wave I } & RT & $1.59 \pm 0.06$ & $1.63 \pm 0.07$ & $1.62 \pm 0.05$ & 2.02 & 0.14 \\
\hline & $\mathrm{LT}$ & $1.58 \pm 0.09$ & $1.62 \pm 0.10$ & $1.60 \pm 0.08$ & 0.95 & 0.39 \\
\hline \multirow{2}{*}{ Wave III } & RT & $3.61 \pm 0.13$ & $3.65 \pm 0.14$ & $3.63 \pm 0.12$ & 0.47 & 0.63 \\
\hline & LT & $3.59 \pm 0.11$ & $3.64 \pm 0.15$ & $3.62 \pm 0.16$ & 0.61 & 0.55 \\
\hline \multirow{2}{*}{ Wave V } & RT & $5.39 \pm 0.20$ & $5.50 \pm 0.17$ & $5.45 \pm 0.21$ & 1.74 & 0.18 \\
\hline & LT & $5.38 \pm 0.22$ & $5.51 \pm 0.18$ & $5.42 \pm 0.19$ & 2.59 & 0.08 \\
\hline \multirow{2}{*}{ I-III } & RT & $2.01 \pm 0.12$ & $2.03 \pm 0.10$ & $2.06 \pm 0.14$ & 0.71 & 0.49 \\
\hline & LT & $2.02 \pm 0.11$ & $2.04 \pm 0.12$ & $2.05 \pm 0.09$ & 0.29 & 0.75 \\
\hline \multirow{2}{*}{ III-V } & RT & $1.79 \pm 0.18$ & $1.84 \pm 0.13$ & $1.81 \pm 0.16$ & 0.59 & 0.56 \\
\hline & LT & $1.75 \pm 0.21$ & $1.82 \pm 0.17$ & $1.77 \pm 0.20$ & 0.81 & 0.45 \\
\hline \multirow{2}{*}{$\mathrm{I}-\mathrm{V}$} & RT & $3.79 \pm 0.16$ & $3.86 \pm 0.15$ & $3.83 \pm 0.19$ & 0.93 & 0.40 \\
\hline & LT & $3.77 \pm 0.22$ & $3.88 \pm 0.17$ & $3.82 \pm 0.18$ & 1.84 & 0.17 \\
\hline
\end{tabular}

*CG: control group.* G1 subjects with music exposur through PLDs with tinnitus group.

*G2: subjects with music exposur through PLDs without tinnitus group.

Table 6: Comparison between control and studied groups as regard to absolute and inter-peak latencies (IPL) of HRR ABR.

\begin{tabular}{|c|c|c|c|c|c|c|}
\hline Latencies & Side & $\begin{array}{c}\mathrm{CG} \\
\mathrm{n}=15 \\
\text { Mean } \pm \mathrm{SD}\end{array}$ & $\begin{array}{c}\mathrm{G} 1 \\
\mathrm{n}=30 \\
\text { Mean } \pm \text { SD }\end{array}$ & $\begin{array}{c}\mathrm{G} 2 \\
\mathrm{n}=15 \\
\text { Mean } \pm \text { SD }\end{array}$ & $\begin{array}{c}\text { ANOVA } \\
\mathrm{F}=\end{array}$ & $P$-value \\
\hline \multirow{2}{*}{ Wave I } & RT & $1.83 \pm 0.10$ & $1.87 \pm 0.11$ & $1.84 \pm 0.12$ & 0.79 & 0.46 \\
\hline & LT & $1.72 \pm 0.14$ & $1.78 \pm 0.15$ & $1.76 \pm 0.13$ & 0.88 & 0.42 \\
\hline \multirow{2}{*}{ Wave III } & RT & $3.88 \pm 0.24$ & $3.92 \pm 0.23$ & $3.86 \pm 0.20$ & 0.39 & 0.67 \\
\hline & $\mathrm{LT}$ & $3.77 \pm 0.22$ & $3.84 \pm 0.13$ & $3.82 \pm 0.17$ & 0.89 & 0.42 \\
\hline \multirow{2}{*}{ Wave V } & RT & $5.83 \pm 0.16$ & $5.88 \pm 0.15$ & $5.87 \pm 0.18$ & 0.50 & 0.61 \\
\hline & LT & $5.76 \pm 0.10$ & $5.81 \pm 0.11$ & $5.80 \pm 0.14$ & 0.95 & 0.39 \\
\hline \multirow{2}{*}{ I-III } & RT & $2.06 \pm 0.16$ & $2.05 \pm 0.17$ & $2.02 \pm 0.15$ & 0.25 & 0.78 \\
\hline & LT & $2.12 \pm 0.21$ & $2.10 \pm 0.24$ & $2.09 \pm 0.22$ & 0.07 & 0.93 \\
\hline \multirow{2}{*}{ III-V } & RT & $1.95 \pm 0.19$ & $1.96 \pm 0.18$ & $2.01 \pm 0.12$ & 0.57 & 0.56 \\
\hline & LT & $1.92 \pm 0.21$ & $1.93 \pm 0.23$ & $1.99 \pm 0.09$ & 0.58 & 0.56 \\
\hline \multirow{2}{*}{$\mathrm{I}-\mathrm{V}$} & RT & $4.00 \pm 0.20$ & $4.01 \pm 0.17$ & $4.06 \pm 0.15$ & 0.55 & 0.58 \\
\hline & LT & $4.04 \pm 0.19$ & $4.03 \pm 0.18$ & $4.05 \pm 0.16$ & 0.07 & 0.94 \\
\hline
\end{tabular}

*CG: control group G1 subjects with music exposur through PLDs with tinnitus.

*G2: subjects with music exposur through PLDs without tinnitus. 
Table 7: Comparison between control and studied groups as regard to speech perception in noise (SPIN) test.

\begin{tabular}{|c|c|c|c|c|c|c|}
\hline SPIN \% & $\begin{array}{c}\mathrm{CG} \\
\mathrm{n}=15 \\
\text { Mean } \pm \text { SD }\end{array}$ & $\begin{array}{c}\mathrm{G} 1 \\
\mathrm{n}=30 \\
\text { Mean } \pm \text { SD }\end{array}$ & $\begin{array}{c}\mathrm{G} 2 \\
\mathrm{n}=15 \\
\text { Mean } \pm \text { SD }\end{array}$ & $\begin{array}{c}\text { ANOVA } \\
\mathrm{F}=\end{array}$ & P-value & Tukey post hoc test \\
\hline RT & $93.6 \pm 3.6$ & $74.9 \pm 3.7$ & $80 \pm 2.5$ & 150.3 & $<0.001$ & $\begin{array}{l}\text { CG vs. G1 }<0.001 \\
\text { CG vs. G2 }<0.001 \\
\text { G1 vs. G2 }<0.001\end{array}$ \\
\hline LT & $93.9 \pm 3.7$ & $75 \pm 3.6$ & $80 \pm 3.5$ & 138.5 & $<0.001$ & $\begin{array}{l}\text { CG vs. } G 1<0.001 \\
\text { CG vs. } G 2<0.001 \\
\text { G1 vs. } G 2<0.001\end{array}$ \\
\hline
\end{tabular}

*CG: control group.

G1 subjects with music exposur through PLDs with tinnitus.

*G2: subjects with music exposur through PLDs without tinnitus .

\section{DISCUSSION}

In the current study, there was no statistical significant difference between study and control groups as regard age and gender (table 1). No statistical significant di $\square$ erences in conventional pure tone audiometry (PTA) between study and control groups (table 2). All had normal hearing thresholds (not exceeding $25 \mathrm{~dB}$ ) at frequency range $(250-8000 \mathrm{~Hz})$ with excellent speech discrimination. Noise exposure duration in the study group ranges from 1 to 4 years. This short duration of exposure may be the reason for absent clinically apparent NIHL in conventional PTAs. Hearing loss arising from exposure to continual loud sounds as in PLD usage is usually gradual and appearing after many years of exposure ${ }^{[20]}$. Extended high frequency audiometry (EHFA) test results:

The current study showed that EHFA was significantly higher at all frequencies in group 1 and group 2 than the controls. There was no statistically significant difference between group 1 and group 2 (table 3 ). These results indicate that EHFA was affected by noise via PLDs not by tinnitus as there was no difference between G1 and G2. These results agree with Peng et al. ${ }^{[21]}$ and Le Prell et al. ${ }^{[22]}$ who carried out research about the risk of damage due to exposure to noise coming from personal music players (PMPs). They found that EHFA had been affected by noise earlier than conventional audiometry and concluded that long-term use of PMPs can impair hearing function. Sulaiman et al. ${ }^{[23]}$ found that when there were no signs of noise induced hearing damage detected on the conventional audiometry $(125 \mathrm{~Hz}$ to $8 \mathrm{kHz}$ ) hearing thresholds at the higher frequencies $(9-16 \mathrm{kHz})$ can be significantly increased. They suggested the presence of an early-stage hearing damage in young PLD users listening for $>1 \mathrm{~h} /$ day and at more than $50 \%$ of the maximum volume settings of their devices. This can be due to initial cochlear damage which may involve lesion to the outer hair cells (OHCs) at the basal end of the cochlea, the area which encodes sounds of higher frequencies that are not tested in conventional audiometry ${ }^{[24]}$.

The present study agree with Barnea et al. ${ }^{[25]}$ who found that extended high frequency audiometry thresholds in tinnitus and non-tinnitus subjects (with normal hearing between $250 \mathrm{~Hz}$ and $8 \mathrm{kHz}$ ) were not significantly different. Also Gilles et al. ${ }^{[10]}$ showed that there was no significant difference in EHFA between noise-exposed subjects with and without tinnitus. They assumed that tinnitus was due to cortical hyperactivity not due to peripheral hearing loss. This was in line with current result between group 1 and group 2 .

However, the current study disagree with some other studies as ${ }^{[14,15,16]}$ who measured EHFA in tinnitus patients with normal hearing between $250 \mathrm{~Hz}$ and $8 \mathrm{kHz}$ and compared their results to those of an age and gender matched controls. They found that most of tinnitus patients had hearing loss for extended high-frequency sounds. Their difference from the current study may be due to different sample size and different age of study group.

\section{Transient Evoked Otoacoustic Emission (TEOAE) test results:}

Signal to noise ratio (SNR) was lower at all frequency bands in group 1 and group 2 than the controls with statistically significant difference (table 4) indicating subclinical cochlear damage. The initial subclinical cochlear damage found in PLD users could progress into permanent NIHL after years of PLD usage.These results agreed with $^{[26-29,17,10,30]}$ who reported that the amplitude of TEOAEs were found to be decreased in regular PLD users compared with non-users.

In this study, there was no statistically significant difference between group 1 and group 2 in TEOAE. In line with our result Gilles et al. ${ }^{[10]}$ reported that TEOAEs 
and DPOAEs did not show significant di $\square$ erences between noise-induced tinnitus and non-tinnitus noise exposed groups. They suggested that the presence of noise-induced tinnitus may occur in the absence of measurable peripheral hearing damage and might cause more central plasticity than expected. The occurrence of tinnitus does not depend upon the degree of damage to the OHCs, but rather upon different neuro-plastic changes that occur in the central auditory system after the same degree of cochlear damage. This may be explained as the neuro-plastic changes in central auditory system induced by deafferentation may vary despite the same amount ofouter hair cells damage and tinnitus may develop in selective cases. Many unknown factors can increase the synchrony of single neuron activities, unmask dormant synapses, create new connections between neurons, or increase the spontaneous activity in central auditory system due to the lack of auditory input ${ }^{[31-33]}$. The current study agreed with $^{[34-35,11]}$ who compared patients suffering from tinnitus and found no difference in TEOAE parameters between patients with tinnitus and normal subjects.

In contrast with results of current study ${ }^{[36,37,38]}$ found that TEOAEs amplitudes were lower in tinnitus patients than non-tinnitus subjects. They concluded that outer hair cell dysfunction might be important in the generation of tinnitus. Therefore, the fact that the TEOAEs had lower amplitudes in the tinnitus group may be related to peripheral hearing disorders that may be the cause of the tinnitus.

\section{Auditory brainstem response $(A B R)$ test results:}

The current study showed no statistically significant difference in absolute and inter-peak latencies of both low and high repetition rate ABR among control and studied groups (table 5,6). ABR used in current study to evaluate the auditory pathway at the brain stem level and results indicate normal neural transmission in auditory nerve in normal hearing adults with and without tinnitus. These results agreed with Gilles et al ${ }^{[10]}$ who did not find any di $\square$ erences in ABR results between tinnitus group and controls (both had normal hearing and exposed to recreational noise).

Also, Barnea et al. ${ }^{[27]}$ performed ABR testing on a tinnitus group with normal hearing sensitivity in the range of $125 \mathrm{~Hz}$ to $8 \mathrm{kHz}$ compared to an age- and gendermatched control group and did not find difference between the groups. Same results were reported by McKee and Stephens ${ }^{[36]}$ and Kehrle et al. ${ }^{[39]}$.

Meanwhile, Gabr ${ }^{[00]}$ reported that there was no significant difference between normal hearing adults with and without tinnitus. However, some tinnitus patients showed abnormal prolonged absolute latencies, IPLs and increased inter-aural latency difference of wave $\mathrm{V}$ (ILD-V). These results suggested impaired neural firing synchronization and transmission in the auditory pathways in tinnitus patients.

Also,Konadath and Manjula ${ }^{[41]}$ reported no significant differences in ABR latency and amplitude between normal hearing subjects with and without tinnitus. However, patients with tinnitus showed abnormally reduced absolute amplitudes of peaks I and V. The reduced amplitude of peaks I and V with normal absolute latencies of peaks I, III and V indicated that the origin of tinnitus may be due to decreased excitation of auditory nerve fibers as a result of peripheral hearing loss in frequencies above $8 \mathrm{kHz}$. This peripheral loss resulted from hair cells damage and could be the reason for reduced output from auditory nerve fibers at the brainstem level. Peripheral damage at the level of the cochlea (damage of OHCs) causes decreased auditory input. Output from central auditory neurons is modulated in response to input alterations ${ }^{[42]}$. The reduced peripheral output leads to sensory deprivation, which leads to altered neural activity in various areas of the brain ${ }^{[43]}$.

\section{Speech perception in noise (SPIN) test results:}

The current study showed highly statistically significant differences in speech perception in noise test (SPIN) between the studied groups and the controls in both ears. Group 1 had lower SPIN scores than control group in both ears (table 7). This means that tinnitus itself could adversely affect speech perception ability in noisy situations in the tinnitus patients in comparison with healthy controls. Possibly suggesting central processes could contribute to tinnitus and centrally located deficits in tinnitus subjects. These results agreed with many studies that measured the speech recognition in noise in tinnitus patients, such as ${ }^{[6,10,44-48]}$, who reported poorer performance on speech perception in noise was seen in tinnitus patients when compared with individuals without complaints of tinnitus. Also, the reason for poorer speech understanding ability in the presence of noise has been attributed to the fact that tinnitus interferes with the speech perception. Also the medial olivary cochlear system had a role in the recognition of auditory stimuli in the presence of noise ${ }^{[49]}$ and a disorder in this system was also hypothesized to cause tinnitus.

In the current study, there was statistically significant difference between G2 and control group (table 7). This result agreed with ${ }^{[32]}$ who measured speech in noise in PLD users.Hrudananda ${ }^{[32]}$ found that subjects who used PLDs had significantly poor speech perception abilities in noise compared to non-users. The observed deterioration in the speech processing abilities in the PLD users, probably due to changes in the central auditory system caused due to prolonged exposure to loud music. It has been reported that long-term noise may have a persistent effect on brain function and behavior, even when the peripheral hearing sensitivity is within normal range ${ }^{[50]}$. It could be said that 
cortical reorganization may occur due to frequent exposure to recreational noise in the absence of any measurable peripheral hearing loss.

In contrast with the current results ${ }^{[49]}$ did not find evidence for speech comprehension impairments. Tugumia et al. ${ }^{[51]}$ used speech-in-Noise test as a validation of an auditory training procedure in 12 subjects suffering from tinnitus. Speech comprehension did not improve due to training and was already in the normal range before training. Reasons for this difference to other studies might be the rather mild condition of the studied patients, their young age and small sample size.

In the current study Group 1 and group 2 had statistically significant difference in SPIN test only with no significant difference in EHFA, TEOAEs or ABR tests. This may be due to tinnitus could develop with a minimal peripheral damage that might not even give rise to any threshold difference or due to adaptive processes of central auditory system (despite the same degree of cochlear damage) that trigger tinnitus ${ }^{[48]}$.

\section{CONCLUSION}

That exposure to recreational noise due to excessive PLDs usage cause elevated EHFA thresholds, decreased TEOAEs amplitudes and decreased SPIN scores despite normal conventional PTA thresholds.

\section{CONFLICT OF INTEREST}

There are no conflicts of interest.

\section{REFERENCES}

1. Levine A. and Oron Y. Tinnitus. Handb ClinNeurol 2015;(129):409-31.

2. Hobson J., Chisholm E. and El Refaie A. Sound therapy (masking) in the management of tinnitus in adults. Cochrane Database Syst Rev. 2012; (11): CD006371.

3. Le'ger C., Moore C. and Lorenzi C. Abnormal speech processing in frequency regions where absolute thresholds are normal for listeners with high-frequency hearing loss. Hear Res 2012; (294):95-103.

4. Le'ger C., Ives T. and Lorenzi C.Abnormal separation of competing speech signals in frequency region where absolute thresholds are normal for listeners with highfrequency hearing loss. Hear Res 2014; (316):102-9.

5. Kalikow N., Stevens N. and Elliott L.Development of a test of speech intelligibility in noise using sentence materials with controlled word predictability. J AcoustSoc Am 1977; (61):1337-51.

6. Newman W., Wharton A., Shivapuja G., et al. Relationships among psychoacoustic judgments, speech understanding ability and selfperceived handicap in tinnitus subjects. Audiology 1994; (33): 47-60.

7. Tyler R. and Baker L. Difficulties experienced by tinnitus sufferers. J Speech Hear Disord $1983 ;$ ( 48) :150-4.

8. Jastreboff J. and Jastreboff M. Tinnitus retraining therapy (TRT) as a method for treatment of tinnitus and hyperacusis patients. J Am AcadAudiol 2000 ; (11):162-77.

9. Punch L., Elfenbein L. and James R.Targeting hearing health messages for users of personal listening devices. Am J Audiol 2011;20(1):69-82.

10. Gilles A., Schlee W., Rabau S., et al. Decreased Speech-In-Noise Understanding in Young Adults with Tinnitus. Front. Neurosci 2016 ; (10):288.

11. Sanchez G., Oliveira C., Kii A., et al. Tinnitus in adolescents: the start of the vulnerability of the auditory pathways. Codas 2015; (27): 5-12.

12. Fernandes L. and dos Santos T. Tinnitus and normal hearing: a study on the transient otoacoustic emissions suppression. Braz J Otorhinolaryngol 2009; 75(3):414-9.

13. Paglialonga A., Bo L., Ravazzani P. et al.,Quantitative analysis of cochlear active mechanisms in tinnitus subjects with normal hearing sensitivity: multiparametric recording of evoked otoacoustic emissions and contralateral suppression. AurisNasus Larynx 2010; (37): 291-8.

14. Shim H., Kim S., Park C., et al. Hearing abilities at ultra-high frequency in patients with tinnitus. ClinExpOtorhinolaryngol; 2009;(2):169-74.

15. Kim K., Park N., Kim M., et al. Prevalence and significance of high-frequency hearing loss in subjectively normal-hearing patients with tinnitus. Annals of Otology, Rhinology \& Laryngology 2011; 120(8):523-8.

16. Gudwani S., Munjal S., Panda N. et al. Correlation of Tinnitus Loudness and Onset Duration with Audiological Profile Indicating Variation in Prognosis. ISRN Otolaryngology 2013; (205714):7 pages.

17. Biassoni E., Serra M., Hinalaf M.,et al. Hearing and 
loud music exposure in a group of adolescents at the ages of 14-15 and retested at 17-18. Noise \& Health 2014; 16 (72) 331-41.

18. Kemp T. Otoacoustic emissions, their origin in cochlear function, and use. $\mathrm{Br}$ Med Bull $2002 ; 63: 223-41$.

19. Tawfik S., Shehata W. and Shalaby A. Development of arabic speech intelligibility in noise test (Arabic SPIN). Ain Shams medical J.1992; 43: 10-12.

20. Fligor J. Personal listening devices and hearing loss: seeking evidence of a long term problem through a successful short-term investigation. Noise Health 2009; 11(44):129-31.

21. Peng H., Tao Z. and Huang W. Risk of damage to hearing from personal listening devices in young adults. J Otolaryngol 2007; 36(3):181-5.

22. Le Prell G., Spankovich C., Lobarinas E. et al. Extended high-frequency thresholds in college students: Effects of music player use and other recreational noise. J Am AcadAudiol 2013;24: 725-39.

23. Sulaiman H., Husain R. and Seluakumaran K. Hearing Risk among Young Personal Listening Device Users: Effects at High-Frequency and Extended High Frequency Audiogram Thresholds. J IntAdvOtol 2015; 11(2): 104-9.

24. Ahmed O., Dennis H., Badran O., et al. Highfrequency $(10-18 \mathrm{kHz})$ hearing thresholds: reliability, and effects of age and occupational noise exposure. Occup Med (Lond) 2001; 51(4):245-58

25. Barnea G., Attias J., Gold S. et al. Tinnitus with normal hearing sensitivity: extended high-frequency audiometry and auditory-nerve brain-stem-evoked responses. Audiology 1990;29:36-45.

26. LePage L. and Murray M. Latent cochlear damage in personal stereo users: a study based on clickevoked otoacoustic emissions. Med J Aust 1998; 169(11-12):588-92.

27. Rosanowski F., Eysholdt U. and Hoppe U. Influence of leisure-time noise on outer hair cell activity in medical students. Int Arch Occup Environ Health 2006; ( 80):25-31.

28. Montoya S., Ibarguen M., Vences R., et al. Evaluation of cochlear function in normal-hearing young adults exposed to MP3 player noise by analyzing transient evoked otoacoustic emissions and distortion products. J Otolaryngol Head Neck Surg 2008; 37(5):718-24.

29. Sulaiman H., Husain R. andSeluakumaran K.Hearing risk associated with the usage of personal listening devices among urban high school students in Malaysia. Public Health; 2013;(127): 710-5.
30. Hrudananda S. Comparison of Post Exposure Effect of Music through Personal Listening Device (PLD) on Hearing in Young Adults. Glob J Otolaryngol 2016; 2(1): 555-76.

31. Bartels H., Staal J. and Albers W.Tinnitus and neural plasticity of the brain. OtolNeurotol 2007; 28:178-84.

32. Roberts E., Eggermont J., Caspary M., et al. Ringing ears: the neuroscience of tinnitus. $\mathrm{J}$ Neurosci 2010; 30: 14972-79.

33. Noreña A. and Farley B. Tinnitus-related neural activity: theories of generation, propagation, and centralization. Hear Res 2013; (295):161-71.

34. Geven L., de Kleine E., Free R. et al. Contralateral suppression of otoacoustic emissions in tinnitus patients. OtolNeurotol 2011; 32(2): 315-21.

35. Mokrian H., Shaibanizadeh A., Farahani S., et al. Evaluation of Distortion and Transient Evoked Otoacoustic Emission in Tinnitus Patients with Normal Hearing. Iranian Journal of Otorhinolaryngology 2014; 26 (1):19-24.

36. McKee J. and Stephens D. An investigation of normally hearing subjects with tinnitus. Audiology 1992;(31): 313-7.

37. Jastreboff J. and Hazell, W.A neurophysiological approch to tinnitus: clinical implications. Br J Audiol 1993;(27):7-17.

38. Granjeiro C., Kehrle M., Bezerra L., et al. Transient and distortion product evoked otoacoustic emissions in normal hearing patients with and without tinnitus. Otolaryngol Head Neck Surg 2008; (138):502-6.

39. Kehrle H., Sampaio A., Granjeiro R., et al. Tinnitus annoyance in normal hearing individuals: correlation with depression and anxiety. Annals of Otology, Rhinology \& Laryngology 2016;125(3): 185-94.

40. Gabr T. Auditory brainstem response audiometry in tinnitus patients. Egyptian Journal of Ear, Nose, Throat and Allied sciences 2011;(12): 115-20.

41. Konadath S. and Manjula P. Auditory brainstem response and late latency response in individuals with tinnitus having normal hearing. Intractable \& Rare Diseases Research 2016; 5(4):262-8.

42. Middleton W., Kiritani T., Pedersen C., et al. Mice with behavioral evidence of tinnitus exhibit dorsal cochlear nucleus hyperactivity because of decreased GABAergic inhibition. ProcNatlAcadSci U S A 2011;(108):7601-06

43. Syka J. Plastic changes in the central auditory system after hearing loss, restoration of function, and during learning. Physiol Rev 2002; (82):601-36. 
44. Huang Y., Lee H., Chung C., et al. Relationships among speech perception, self-rated tinnitus loudness and disability in tinnitus patients with normal puretone thresholds of hearing. ORLJ. Otorhinolaryngol. Relat. Spec 2007; (69): 25-29.

45. Hennig R., Costa J., Urnau D., et al..Recognition of speech of normal-hearing individuals with tinnitus and hyperacusis. Int. Arch. Otorhinolaryngol 2011; (15): 21-8.

46. Ryu S., Ahn H., Lim W., et al. Evaluation of masking $\mathrm{e} \square$ ects on speech perception in patients with unilateral chronic tinnitus using the hearing in noise test. Otol. Neurotol 2012;(33): 1472-76.

47. Jain C. and Sahoo P. The effect of tinnitus on some psychoacoustical abilities in individuals with normal hearing sensitivity. Int. Tinnitus J. 2014; (19): 28-35.

48. Moon J., Won H., Kang W., et al. Influence of tinnitus on auditory spectral and temporal resolution and speech perception in tinnitus patients. J. Neurosci 2015; (35): 14260-69.

49. Grataloup C., Hoen M., Veuillet E., et al. Speech restoration: an interactive process. J Speech Lang Hear Res 2009; 52(4):827-38.

50. Kujawa S. and Liberman M. Adding insult to injury: cochlear nerve degeneration after "temporary" noise-induced hearing loss. J. Neurosci 2009; (29): 14077-85.

51. Tugumia D., Samelli G., Matas G., et al. Auditory training program in subjects with tinnitus. Codas 2016;(28): 27-33. 